\title{
Wernicke's encephalopathy in a patient with severe acute pancreatitis after cholecystectomy and exploration of biliary tract
}

Huihui Du

Central South University

Xiaohua Lei

Central South University

Qiang Liu

Central South University

Xundi Xu ( $\square$ xuxundi@csu.edu.cn )

Central South University https://orcid.org/0000-0003-3686-2975

\section{Case Report}

Keywords:

Posted Date: November 30th, 2018

DOI: https://doi.org/10.21203/rs.2.45/v1

License: (c) (i) This work is licensed under a Creative Commons Attribution 4.0 International License. Read Full License 


\section{Abstract}

Background: Wernicke's encephalopathy (WE) is a disease classically associated with thiamine deficiency『and generally manifests with the triad of opthalmopathy, ataxia and confusion. Only about $20 \%$ of the cases can be defined from the relevant literature, with the rest unnoticed and thus unreported. Here, we would like to report a case of a patient with severe acute pancreatitis who suffered from Wernicke's encephalopathy after cholecystectomy and exploratory of biliary tract.

Case presentation: A 37-year-old Chinese male patient diagnosed with severe acute pancreatitis was admitted to our institute's hospital for further treatment. The patient had a 25-days history of abdominal pain as well as 8-days emesis, and wasn't addicted in alcohol consumption. He did not receive any regular physical examination or treatment. One months before cholecystectomy and exploratory of biliary tract $囚$ he was observed to have abdominal pain ,poor appetite, emesis and sclera icterus .He was referred to the local hospital, received supportive treatment, including antibiotics, parenteral nutrition and liverprotection drugs. However, he got worse after those treatment $₫$ so transferred from the local hospital to our hospital for further therapy. After operation he was admitted in general ward for further treatment. When his vital signs and laboratory examination came to stability. He recovered well with supportive treatments, including antibiotics, liver-protection, protecting gastric mucosa and so on. However, his oral intake was too low to support himself, and he was singly dependent on total parenteral nutrition without vitamin B1. On the 7th post-operative day, psychiatric and neurological disorders appeared without an obvious cause, symptoms including babbling, irritability, apathy, non-purposeful movements, and dystaxia. On the basic of his clinical features and brain magnetic resonance imaging scan results, Wernicke encephalopathy was eventually diagnosed.

Conclusions: Although extremely rare, this index patient highlights that Wernicke's encephalopathy could be associated with severe acute pancreatitis. This is very helpful for the therapeutic implications and evaluating curative effect. Thiamine should be provided for patients who have a surgery of cholecystectomy and exploratory of biliary tract so as to prevent and cure Wernicke's encephalopathy.

\section{Background}

Wernicke's encephalopathy (WE) is a neuropsychiatric syndrome resulting from thiamine (vitamin B1) deficiency and occurs frequently in patients with prolonged parenteral nutrition and reduced oral intake[1].The development of WE after parenteral nutrition due to the lack of thiamine was first reported in 1975 by Blennow[2]. The classical triad of WE includes opthalmopathy, ataxia and confusion. And delay in its diagnosis may result in severe irreversible neurological damage, or even death. Only about $20 \%$ of the cases can be defined from the relevant literature, with the rest unnoticed and thus unreported[3]. Although early diagnosis and treatment with thiamine can observably reverse the symptoms, the mortality rate of Wernicke's encephalopathy (WE) still remains $10 \%-20 \%$ due to misdiagnosis[4]. 
Although it is most frequently seen due to chronic malnutrition associated with long term alcohol use, it may occur in patients who suffer from severe acute pancreatitis and have a cholecystectomy and exploratory of biliary tract.

Here, we present a nonalcoholic male patient with severe acute pancreatitis who developed Wernicke encephalopathy after cholecystectomy and exploratory of biliary tract. This case emphasizes the need for early diagnosis and thiamine supplementation.

\section{Case presentation}

A 37-year-old Chinese male patient diagnosed with severe acute pancreatitis was admitted to our institute's hospital for further treatment. The patient had a 25-days history of abdominal pain as well as 8-days emesis, and wasn't addicted in alcohol consumption. He did not receive any regular examines or treatment. One months before cholecystectomy and exploratory of biliary tract $₫$ he was observed to have abdominal pain ,poor appetite, emesis and sclera icterus. He was referred to the local hospital, his cardiovascular and neurological exams were normal. His vital signs were: heart rate of $117 \mathrm{~min}-1$, blood pressure $126 / 82 \mathrm{mmHg}$, temperature $38.2^{\circ} \mathrm{C}$, and respiratory rate $29 \mathrm{~min}-1$.Serum amylase was $723 \mathrm{IU} / \mathrm{L}$ (reference range: $25-125 \mathrm{IU} / \mathrm{L}$ ) and lipase $554 \mathrm{IU} / \mathrm{L}$ (reference range: $23-300 \mathrm{IU} / \mathrm{L}$ ). The diagnosis of severe acute pancreatitis was confirmed after an abdominal CT scan showed biliary sludge in the gallbladder and a large fluid collection around a severely inflamed pancreas. APACHE-II scores for the patient reached 13 points. Supportive treatment, including antibiotics $\$ parenteral nutrition and liver-protection were performed. However, the treatment took a change for the worse, so the patient transferred from the local hospital to our hospital for a surgery. After operation, he was admitted in general ward for further treatment. When his vital signs and laboratory examination came to stable. He recovered well with supportive treatments, including antibiotics, liver-protection, protecting gastric mucosa and so on. However his oral intake was too low to support himself, and he was singly dependent on total parenteral nutrition without vitamin B1. On the 7th post-operative day, psychiatric and neurological disorders appeared without an obvious cause, symptoms including babbling, irritability, apathy, non-purposeful movements, and dystaxia. In addition, there were no obviously infectious signs. He had no history of chronic alcohol consumption, psychosis, diabetes mellitus, hypertensive disease, coronary artery disease, or tuberculosis. The laboratory evaluation yielded the following: white blood cell count, $12.6 \times 109 / \mathrm{L}$; neutrophil percentage, $87.20 \%$; red blood cell count,4.65 $\times 109 / \mathrm{L}$; platelet count, $110 \times 109 / \mathrm{L}$; hemoglobin, $159 \mathrm{~g} / \mathrm{L}$; international normalized ratio, 1.74; blood ammonia, 29.2umol/L; serum alanine aminotransferase, $172.3 \mathrm{U} / \mathrm{L}$; serum aspartate aminotransferase, $60.4 \mathrm{U} / \mathrm{L}$; serum total protein, $84.7 \mathrm{~g} / \mathrm{l}$; serum albumin, $51.2 \mathrm{~g} / \mathrm{l}$; serum sodium, $133 \mathrm{mmol} / \mathrm{L}$; and serum kalium, $4.0 \mathrm{mmol} / \mathrm{L}$; serum calcium 2.75 $\mathrm{mmol} / \mathrm{l}$. Therefore, brain magnetic resonance imaging (MRI) was performed(Figure1). On the basic of his clinical manifestations and brain magnetic resonance imaging scan results, Wernicke encephalopathy was eventually diagnosed. After intravenous vitamin B1 prescribed, with $500 \mathrm{mg}$ daily for the first 3 days and $100 \mathrm{mg}$ daily for the second 3 days, all symptoms were resolved apparently and returned to normal in the next week[5]. Follow-up of the patient was done 3 months later after operation, MRI shows no obvious abnormality in the lesion and his symptoms returned to normal. 


\section{Discussion And Conclusion}

As it is well-known, active form thiamine pyrophosphate plays an important role in the pentose phosphate and the tricarboxilic acid cycle which are essential in the metabolism of carbohydrates and lipids. Active form thiamine pyrophosphate also play an important part in energy metabolism such as transketolase, pyruvate dehydrogenase, and alpha ketoglutarate dehydrogenase. Lack of thiamine, the excessive metabolism of brain cell will suffer from cell energy deficit, acidosis and even cellula death[6]. Since the body's reserve of thiamine is exhausted after nearly 20 days of scant supplementation, thiamine deficiency may happen within as few as 18-20 days in patients receiving severe thiamine-free diets. And the thiamine increases in demand in ill patients.

Wernicke encephalopathy is an universally known neurological complication of thiamine deficiency that mostly occurs in alcoholic persons and is regarded as a rare complication of severe acute pancreatitis. As is presented, doctors will take WE into account when there is the presence of the classical triad of Wernicke encephalopathy including opthalmopathy, ataxia and confusion. However, Wernicke encephalopathy might be underdiagnosed, even misdiagnosed because there usually be non-specific clinical presentation and about $19 \%$ of patients have none of the symptoms of the specific triad[7]. There are few case reports in patients with Wernicke encephalopathy who suffer from severe acute pancreatitis and receive a surgery of cholecystectomy and exploratory of biliary tract.

In our case report, the patient did not manifest the complete triad and the most obvious symptoms and signs were mental status changes. The systematic clues to this disease are anorexia, confusion, blindness, amyasthexia, nystagmus, irritability and recurring vomiting episodes[8]. From the typical clinical manifestation, Wernicke encephalopathy could be diagnosed primarily[9]. The differential diagnosis between Wernicke encephalopathy and other psychiatric and/or neurological disorders such as pancreatic encephalopathy is extremely significant[10]. Pancreatic encephalopathy is a complication of severe acute pancreatic which manifest as confusion, restlessness, altered mentality, and there are diffuse demyelination and white matter changes in cerebral imaging[11]. Although different pathophysiological mechanisms such as altered hemodynamic, metabolic status, electrolyte and fluid disorders, inflammatory cytokines, and a direct neurotoxic action of phospholipase were put forward, the physiopathology is not completely understood[11]. It is difficult to distinct the difference between Wernicke encephalopathy and pancreatic encephalopathy due to the overlap of neuropsychiatric manifestations, the multiple concomitant clinical and metabolic alterations capable of explaining the neurologic alterations. Regarding the difficulties, some physicians who joined the discussion still insisted on their initial diagnosis of pancreatic encephalopathy and they did not give up their opinions until the condition of the patient was recovered by intravenous by vitamin B1. Therefore, when difficulties in distinguishing between Wernicke encephalopathy and other psychiatric and/or neurological disorders appear, intravenous vitamin B1 could be regarded as a discriminative method or a preemptive treatment.

The more time waste before WE is timely and correctly diagnosed, the more dangerous the patient is. The most valuable method to diagnose Wernicke encephalopathy is brain magnetic resonance imaging, with 
a high(93\%) specificity. However, due to poor sensitivity(53\%), it is not reliable enough for a normal magnetic resonance imaging to exclude the disease[12]. The typical magnetic resonance imaging findings of WE include symmetrically increased T2 signal in the thalami, mammillary bodies, tectal plate, and periaqueductal gray matter. And other changes could also be seen in MRI, such as abnormal signalintensity in the cerebellum, the cerebellar vermis, the cranial nerve nuclei, the dentate nuclei, the red nuclei, the caudate nuclei, the corpus callosum, and the cerebral cortex[13, 14].

Wernicke's encephalopathy can emerge in post-operative patients who have prolonged total parenteral nutrition without given enough thiamine supplementation, which make the addition of thiamine indispensable. It is reported that delay in treatment with thiamine before the magnetic resonance imaging findings can lead to progressive and irreversible damage and even death. So early parenteral suplement with thiamine after cholecystectomy and exploratory of biliary tract can quickly reverse symptoms and prevent further injury[15].

In conclusion, human beings may develop Wernicke's encephalopathy at any age and not rarely in person who suffers from severe acute pancreatitis after cholecystectomy and exploratory of biliary tract. And Wernicke's encephalopathy should be taken into consideration when patients with prolonged total parenteral nutrition present one or more symptoms of opthalmopathy, ataxia and altered mentality. The most valuable examination for WE is brain magnetic resonance imaging. After cholecystectomy and exploratory of biliary tract, a multivitamin and thiamine should be provided for patients so as to prevent and cure Wernicke's encephalopathy[16].

\section{Declarations}

\section{Ethics approval and consent to participate}

Written informed consent was obtained from the patient for publication of this Case report and any accompanying images. A copy of the written consent is available for review by the Editor of this journal. This case report study was carried out respecting the Declaration of Helsinki in its current version. Ethical approval was approved by the ethics committee of the the Second Xiangya Hospital.

\section{Consent for publication}

Signed informed consents were obtained from the patient for publication of this Case Report and any accompanying images. A copy of the consent form is available for review by the Editor of this journal.

\section{Availability of data and material}

All data generated during the current study are available from the corresponding author in reasonable request.

\section{Competing interests}


The authors declare that they have no competing interests.

\section{Funding}

This work was supported by the National Natural Science Foundation of China (No, 81670111). The funding paid staff costs for the design of the study and collection, and analysis of data and in writing the manuscript.

\section{Authors' contributions}

$\mathrm{HHD}$ contributed to the concept, drafting and reporting of the case. $\mathrm{XHL}$ and QL contributed the reporting and revising the report. XDX contributed to the analysis and interpreting the results and revising the report. All authors read and approved the final manuscript. All authors read and approved the contents of the case report.

Acknowledgements

The authors thank the family and patient for their participation to this study.

\section{References}

1. Day GS, del Campo CM: Wernicke encephalopathy: a medical emergency. CMAJ : Canadian Medical Association journal = journal de l'Association medicale canadienne 2014, 186(8):E295.

2. Blennow G: Wernicke encephalopathy following prolonged artificial nutrition. American journal of diseases of children (1960) 1975, 129(12):1456.

3. Azim W, Walker R: Wernicke's encephalopathy: a frequently missed problem. Hospital medicine (London, England : 1998) 2003, 64(6):326-327.

4. Sechi G: Thyrotoxicosis-associated Wernicke's encephalopathy. Journal of general internal medicine 2008, 23(6):897.

5. Boulanger AS, Paquette I, Letourneau G, Richard-Devantoy S: [Wernicke encephalopathy: Guiding thiamine prescription]. L'Encephale 2017, 43(3):259-267.

6. Thomson AD, Marshall EJ: The natural history and pathophysiology of Wernicke's Encephalopathy and Korsakoff's Psychosis. Alcohol and alcoholism (Oxford, Oxfordshire) 2006, 41(2):151-158.

7. Sechi G, Serra A: Wernicke's encephalopathy: new clinical settings and recent advances in diagnosis and management. The Lancet Neurology 2007, 6(5):442-455.

8. Sadighi Z, Sabin ND, Hayden R, Stewart E, Pillai A: Diagnostic Clues to Human Herpesvirus 6 Encephalitis and Wernicke Encephalopathy After Pediatric Hematopoietic Cell Transplantation. Journal of child neurology 2015, 30(10):1307-1314. 
9. Weidauer S, Nichtweiss M, Lanfermann H, Zanella FE: Wernicke encephalopathy: MR findings and clinical presentation. European radiology 2003, 13(5):1001-1009.

10. Sun GH, Yang YS, Liu QS, Cheng LF, Huang XS: Pancreatic encephalopathy and Wernicke encephalopathy in association with acute pancreatitis: a clinical study. World journal of gastroenterology 2006, 12(26):4224-4227.

11. Zhang XP, Tian H: Pathogenesis of pancreatic encephalopathy in severe acute pancreatitis. Hepatobiliary \& pancreatic diseases international : HBPD INT 2007, 6(2):134-140.

12. Antunez E, Estruch R, Cardenal C, Nicolas JM, Fernandez-Sola J, Urbano-Marquez A: Usefulness of CT and MR imaging in the diagnosis of acute Wernicke's encephalopathy. AJR American journal of roentgenology 1998, 171(4):1131-1137.

13. Nolli M, Barbieri A, Pinna C, Pasetto A, Nicosia F: Wernicke's encephalopathy in a malnourished surgical patient: clinical features and magnetic resonance imaging. Acta anaesthesiologica Scandinavica 2005, 49(10):1566-1570.

14. Manzo G, De Gennaro A, Cozzolino A, Serino A, Fenza G, Manto A: MR imaging findings in alcoholic and nonalcoholic acute Wernicke's encephalopathy: a review. BioMed research international 2014, 2014:503596.

15. Latt N, Dore G: Thiamine in the treatment of Wernicke encephalopathy in patients with alcohol use disorders. Internal medicine journal 2014, 44(9):911-915.

16. Galvin R, Brathen G, Ivashynka A, Hillbom M, Tanasescu R, Leone MA: EFNS guidelines for diagnosis, therapy and prevention of Wernicke encephalopathy. European journal of neurology 2010, 17(12):14081418.

\section{Figures}



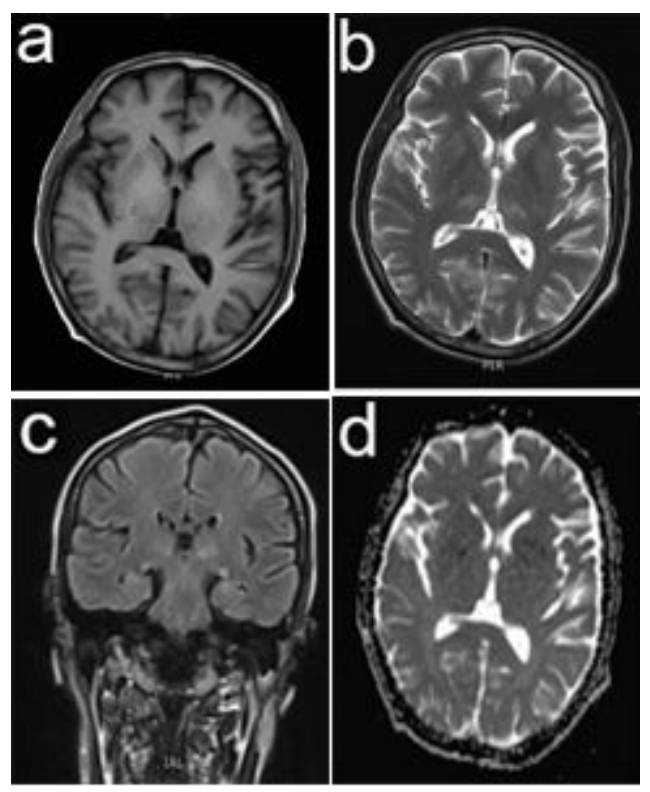

Figure 1

Brain magnetic resonance imaging demonstrating that there were symmetrically increased T2 signals in the medial parts of the thalamus. (a-b) magnetic resonance imaging shows long T1, long T2 in the medial parts of the thalamus. (c) There were high signals in the medial parts of the thalamus and pons. (d) There were high signals in the medial parts of the thalamus.

\section{Supplementary Files}

This is a list of supplementary files associated with this preprint. Click to download.

- supplement1.docx 